Katrin Bicher* und Barbara Wiermann

\title{
Normdaten zu „Werken der Musik“ und ihr Potenzial für die digitale Musikwissenschaft
}

https://doi.org/10.1515/bfp-2018-0043

Zusammenfassung: Der Aufsatz diskutiert Normdaten zu musikalischen Werken in der GND und erörtert Möglichkeiten ihrer (Nach-)Nutzung in bibliothekarischen und musikwissenschaftlichen Kontexten. Herausforderungen sowohl des theoretischen Konzepts als der praktischen Umsetzung werden benannt und das Potenzial der in den Normdaten enthaltenen Informationen wird aufgezeigt.

Schlüsselwörter: Normdaten; musikalische Werke; Bibliothekskatalog; digitale Musikwissenschaft

\section{Authority Files for Musical Works and their Potential for Digital Musicology}

Abstract: The paper discusses conceptual matters and implementation practices for authority information for musical works in the German authority file (GND). Options of re-use of the authority data in libraries as well as in musicological contexts are introduced. Challenges of theoretical concepts as well as practical realization are shown and potentials of information included in authority files are demonstrated.

Keywords: Authority files; musical works; library catalog; digital musicology

\section{Inhalt}

1 Normdaten und „Werke der Musik“ in der Gemeinsamen Normdatei . . . . . . . . . . . . . . . . . . 223

2 Potenzial musikalischer Werk-Normdaten . . . . 230

3 Fazit ...................... 235

Das Chalumeau ist ein Instrument des 18. Jahrhunderts. Es gehört zur Familie der Rohrblattinstrumente und gilt als Vorgänger der Klarinette. Insofern überrascht nicht, dass es in den Besetzungshinweisen zu Musik des 18. Jahrhunderts etwa bei Bononcini, Graupner oder Telemann auftaucht.

*Kontaktperson: Katrin Bicher, katrin.bicher@slub-dresden.de Dr.Barbara Wiermann, barbara.wiermann@slub-dresden.de
Aber ist es tatsächlich auf den engen zeitlichen Rahmen Mitte des 18. Jahrhunderts und den österreichisch-deutschen Raum beschränkt? Wird es auch von Komponisten anderer Regionen eingesetzt oder erlebt es eine Renaissance beispielsweise in der Musik des 19. oder 20. Jahrhunderts? Für den Einzelfall vermag ein Blick in die zugehörigen Quellen Fragen dieser Art zu beantworten. Eindrücke von strukturellen Bewegungen der Geschichte aber bedürfen des größeren Abstands, um Muster und gegebenenfalls Entwicklungen erkennen zu können. Und da sich unser Bild der Geschichte bekanntlich sowohl aus der auf das Individuelle blickenden Historischen Anthropologie im Sinne einer Alltagsgeschichte als auch einer verallgemeinernden Strukturgeschichte ergibt, hießen die obigen Fragen in ihrer konsequenten Fortsetzung dann: Erlebten bestimmte Gattungen in bestimmter Besetzung komponiert von Künstlern bestimmter Regionen aber uraufgeführt in ganz anderen geografischen Kontexten besondere Moden? Eine derartige Kombination von Aspekten musikalischer Werke mag zunächst vielleicht abwegig erscheinen. Aber die für die Beantwortung nötigen Informationen liegen nicht nur bereit, sondern sind in zunehmendem Maße als Meta-Daten zu den historischen Phänomenen gezielt adressier- und analysierbar und versprechen so neue Perspektiven auf die Musikgeschichte. ${ }^{1}$

Einen für (musik-)wissenschaftliche Fragen bisher noch kaum genutzten Pool solcherart Informationen bieten Normdateien, wie sie in bibliothekarischen Kontexten in Deutschland seit einigen Jahrzehnten zentral aufgebaut und heute in der Gemeinsamen Normdatei (GND) der Deutschen Nationalbibliothek gepflegt werden. Vor allem Personendaten und Geografika der GND haben inzwischen über die Grenzen des Bibliothekswesens hinaus eine weite Akzeptanz und Verwendung erfahren. ${ }^{2}$ Als für die Musikwissenschaft besonders interessant und relevant können sich die in der GND enthaltenen normierten Datensätze zu „Werken der Musik“ erweisen. Im Folgenden soll dieser Datenkorpus deshalb charakterisiert und genauer auf bi-

1 Vgl. dazu z.B. Moretti, Franco: Distant Reading, Konstanz 2016. 2 Etwa als Referenz für Entitäten in Lexika oder Editionen, vgl. Stadler, Peter: Normdateien in der Edition. In: editio 26 (2012), 174-83, DOI: 10.1515/editio-2012-0013. 
bliothekarische als auch außerbibliothekarische und explizit wissenschaftliche Nutzungsmöglichkeiten hin befragt werden.

\section{Normdaten und „Werke der Musik" in der Gemeinsamen Normdatei}

Das strukturierte Ablegen von Informationen zum schnellen Wiederauffinden ist essentiell für Bibliotheken als Ort des sortierten Wissens. Mithilfe von Thesauri werden hier deshalb nicht nur die Informationen selbst geordnet, sondern auch Informationen über Informationen systematisiert, wird Identisches begrifflich zusammengefasst und schnell recherchierbar gehalten. Vor allem für den Bereich von Personen und Orten, deren Bezeichnungen im originalen Zusammenhang oft differieren, die aber für die Beschreibung von Medien auf ihrer Metadatenebene substantiell sind, entstand durch eine Normung und das Zurückführen der Bezeichnung auf eine jeweils gleiche Zeichenkombination ein System, das verschiedene Benennungen zuverlässig dem gleichen Sachverhalt zuordenbar hält. Das in einer solchen Normdatei abgelegte Datum gilt damit als Referenz für alle individuellen Ausprägungen desselben ,Umstandes‘. So erleichtert eine Normdatei die Katalogisierung von Entitäten in den individuellen Systemen, sie ist zugleich - als gemeinsamer Bezugspunkt mit der Notwendigkeit absoluter Integrität und Verlässlichkeit im Sinne von Zitierbarkeit, Datenpersistenz und Qualitätssicherung - aber auch Prüfstein und Garant für die Konsistenz der Daten. Während in lokalen projektspezifischen Normdateien konkrete auf die jeweiligen Inhalte und Aufgaben bezogene Daten vorgehalten werden, meist über eigene Ontologien verwaltet und verknüpft und oft nur einem kleinen Nutzerkreis zugänglich, bieten globale Normdateien durch einfache Strukturen ein hohes Maß an Anschlussfähigkeit.

In Deutschland wurden 2012 die bis dahin separat gehaltenen nationalen Normdateien für Personen, Körperschaften und Sachbegriffe in der GND zusammengefasst. ${ }^{3}$ Zur selben Zeit wurden die im Deutschen Musikarchiv vor-

3 Die GND enthält im Wesentlichen sechs Entitäten: Personen, Körperschaften, Konferenzen/Veranstaltungen, Geografika, Sachbegriffe und Werke. Gepflegt wird sie gemeinschaftlich u.a. von der DNB und den Bibliotheksverbünden, http://www.dnb.de/DE/Standardisierun g/GND/gnd_node.html. gehaltenen Daten zu Einheitssachtiteln der Musik (EST) in die GND integriert. ${ }^{4}$

Den theoretischen Hintergrund für das System der nationalen Normdateien bilden die von der IFLA erarbeiteten Functional requirements for bibliographic records $(\mathrm{FRBR})^{5}$ und die Functional requirements for authority data $(\text { FRAD })^{6}$, die die hier angelegten Entitäten, ihre Verhältnisse untereinander und ihre bestimmenden Merkmale definieren und damit einen internationalen Datenaustausch ermöglichen. ${ }^{7}$ Das FRBR-Modell nimmt dabei (abstrakte) Werke an, die (noch nicht materialisierte) Expressionen hervorbringen können. Diese Expressionen verkörpern sich in Manifestationen, von denen wiederum einzelne Exemplare existieren. Außerdem enthält es Personen und Körperschaften und schließlich Konzepte, Gegenstände, Ereignisse und Orte, jeweils mit Relationen zu den beiden anderen Entitätengruppen. Hinzu kommen Merkmale - eigentlich wiederum verkürzte Beziehungen -, die die einzelnen Entitäten ausprägen können, wie Daten und Orte (Abb. 1). ${ }^{8}$

Das FRBR-Modell, zunächst und in erster Linie theoretischer Hintergrund für konkrete bibliografische (Titel-) Metadaten, liegt auch den abstrahierenden Normdaten zu Grunde. ${ }^{9}$ Nach den FRAD wird das Werk als Abstraktum, wie es in der obersten Ebene der FRBR-Entitätengruppe 1 verankert ist, durch die Merkmale Form of work, Date of work, Medium of performance, Subject of the work, Numeric designation, Key, Place of origin of the work, History,

4 Die letzte gedruckt veröffentlichte Ausgabe der Einheitssachtiteldatei des Deutschen Musikarchivs Berlin war die Ausgabe 6, 2004 mit Redaktionsschluss 17. August 2004.

5 IFLA (Hrsg.): Functional Requirements for Bibliographic Records. Korrigierte und ergänzte Fassung o.O. 2009.

6 IFLA Working Group on Functional Requirements and Numbering of Authority Records (Hrsg.): Functional Requirements for Authority Data - A Conceptual Model. Korrigierte und ergänzte Fassung o.O. 2013 (https://www.ifla.org/files/assets/cataloguing/frad/frad_2013. pdf); Deutsche Übersetzung der Version von 2009: Patton, Glenn E. (Hrsg.): Funktionale Anforderungen an Normdaten. Berlin/New York 2010.

7 Eine Zusammenführung der Daten verschiedener nationaler Normdateien bietet die übergeordnete Metanormdatei Virtual International Authority File (VIAF), http://www.oclc.org/en/viaf.html, vgl. auch Hengel, Christel: Normdaten und Metadaten. Die Idee eines Internationalen Authority File. In: Zeitschrift für Bibliothekswesen und Bibliographie 50 (4) (2003), 210-14.

8 Zur Erweiterung des Modells zu FRBR-oo (object oriented) vgl. IFLA (Hrsg.): Definition of FRBRoo - A Conceptual Model for Bibliographic Information in Object-Oriented Formalism. Den Haag 2015.

9 Die FRAD als grundsätzliches Modell für Normdaten sehen zwar auch die FRBR-Entitäten Manifestation und Exemplar vor - für die Verwendung in der GND, die keine physischen Materialen verzeichnet, sind diese Ebenen eines Werkes jedoch nicht relevant. 


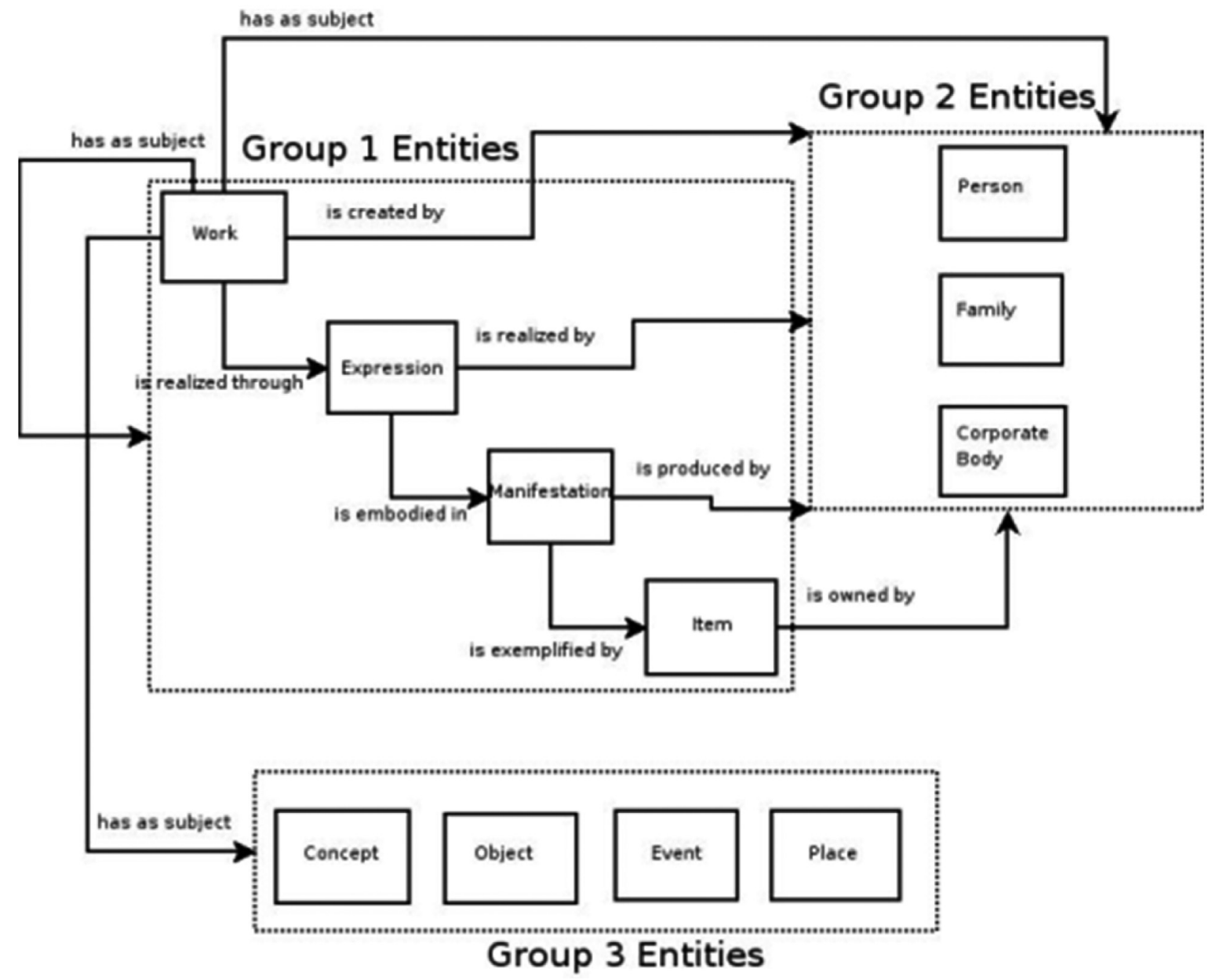

Abb. 1: Barbara Tillett: FRBR-Modell nach https://cataids.wordpress.com/2009/06/24/frbr-do-you-understand-it/

Other distinguishing information bestimmt und disambiguiert, ${ }^{10}$ die in der GND mit folgenden Datenfeldern umgesetzt werden: ${ }^{11}$

\begin{abstract}
Identifikator
Satzart bzw. Satztyp
\end{abstract}

Katalogisierungsquelle

10 IFLA (Anm. 6) 20.

11 Die Ansicht im DNB-Katalog stellt nicht alle erfassten Felder dar. Über die Marc21-XML-Repräsentation jedoch erhält man Einsicht in alle Datenfelder, die entsprechenden Analysen zu Grunde gelegt werden.

12 Der Satztyp „wie“ bezieht sich auf Expressionen zu Werken unabhängig ihrer Disziplin und Medialität - also Musikwerken ebenso wie literarischen oder Werken der bildenden Kunst.
Bevorzugter Titel

Abweichender Titel Ausdifferenzierung der Satzart nach dem Inhalt und der FRBREbene (musikalisches Werk: „wim“, Expression: „wie“12) der Erfassung zu Grunde gelegtes Regelwerk

Beziehung zu Personen/ Körperschaften

Beziehung zu einem Werk 


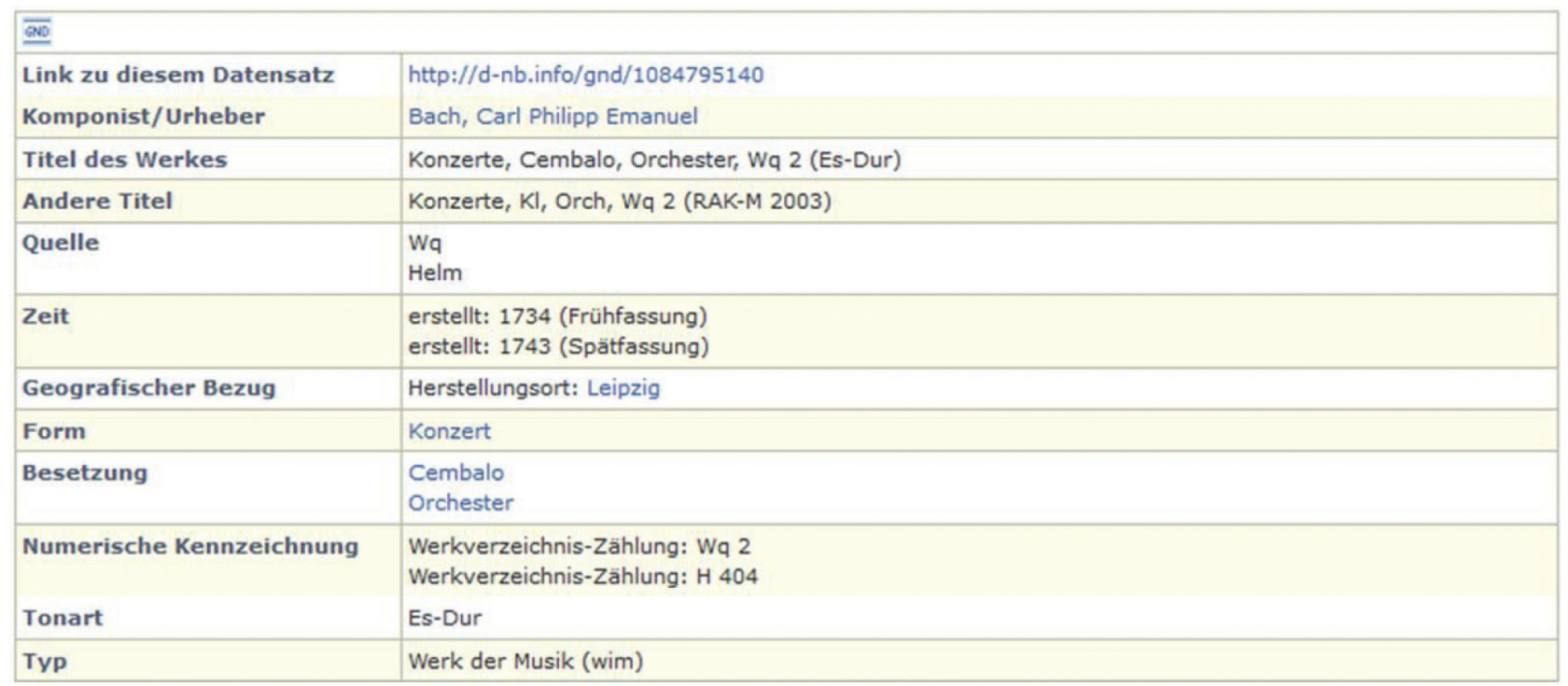

Abb. 2: GND-Datensatz zu Carl Philipp Emanuel Bach: Konzert für Cembalo und Orchester Es-Dur, http://d-nb.info/gnd/1084795140

Beziehung zu einem Geografikum Verknüpfung mit Angaben der FRBR-Gruppe 1 (als Merkmal des Werkes wie in Abb. 2) oder der FRBR-Gruppe 3 (als thematischer Bezug wie in Abb. 4)

Form des Musikwerks

Besetzung

Numerische Bezeichnung des Musikwerks

Tonart des Musikwerks

Datum des Musikwerks

Sprache der ersten Expression

Quellenangabe

Ländercode

GND-Systematik

Ergänzende Angaben
Gattung / Genre / Form als Merkmale von Entitäten der FRBRGruppe 1

Besetzung und Besetzungsstärke als Merkmale von Entitäten der FRBR-Gruppe 1

Werkverzeichnis-, Opus- oder andere Zählungen

Als Merkmal von Entitäten der FRBR-Gruppe 1

Verschiedene mit dem Werk in Verbindung stehende Daten wie Entstehung, Uraufführung etc.

Sprache des Werkes

Angabe, woher die Informationen stammen

Angabe zur Herkunft des Verfassers (in den gegenwärtigen Grenzen) oder - bei unbekanntem Urheber - des zum Werk in einer Beziehung stehenden Landes

Angabe der Systematikstelle innerhalb der GND-Klassifikation

Biografische, historische und andere Angaben
Außerdem werden Metadaten der Erfassung gesichert (Namen, Zeiten etc. der Erstellung/Änderung der Datensätze).

Das von FRBR und FRAD definierte System liefert dabei nicht zuletzt durch die enthaltenen Querverweise ein Netz von Informationen, mit denen verschiedene Wissensräume erschlossen werden und die eingangs gestellte Frage nach der Bedeutung des Chalumeaus ohne weitergehende Quellenstudien auf den ersten Blick leicht beantwortbar zu sein scheint. Realiter jedoch ergeben sich einige, zum Teil historisch bedingte, praktische und konzeptionelle Herausforderungen.

Die Normdatensätze zu musikalischen Werken, wie sie seit 2012 in der GND geführt werden, entstammen im Wesentlichen zwei verschiedenen Quellen: der ehemaligen EST-Datei des Deutschen Musikarchivs (DMA) und der Schlagwortnormdatei. Mit derzeit etwa 178000 Datensätzen ist, wohl bedingt durch das junge Alter dieses Entitätentyps, ihre Anzahl überschaubar. Ihre Qualität ist aufgrund der Entstehungsgeschichte der Werkdatei äußerst heterogen. Nur ein geringer Teil der Daten ist nach dem 2014 eingeführten Regelwerk Resource Description and Access (RDA) entstanden. Da zudem die RDA zahlreiche Merkmale als optional beschreiben bzw. nur verlangen, sofern Titel und Urheber nicht zur eindeutigen Identifizierung ausreichen, erfüllt nur ein noch kleinerer Teil der Daten das vollständige FRAD-Schema. Neben diesen eher redaktionellen Punkten aber sind auch konzeptionelle Fragen (noch) nicht gelöst resp. werden aus dem FRBR-Modell (noch) nicht konsequent in der GND umgesetzt. So gilt es beispielsweise, die Anwendbarkeit der im FRBR-Modell definierten Vierstufigkeit der ersten Entitätengruppe - al- 


\begin{tabular}{|l|l|}
\hline $\begin{array}{l}\text { Link zu diesem Datensatz } \\
\text { Komponist/Urheber }\end{array}$ & $\begin{array}{l}\text { http://d-nb.info/gnd/300157800 } \\
\text { Stravinsky, Igor }\end{array}$ \\
\hline Titel des Werkes & The flood \\
\hline Beteiligt & Librettist: Craft, Robert \\
\hline Andere Titel & $\begin{array}{l}\text { Die Flut } \\
\text { Le déluge } \\
\text { Il diluvio }\end{array}$ \\
\hline Quelle & StravinskyWV \\
\hline Erläuterungen & Definition: ein musikalisches Spiel \\
\hline Zeit & 1961 -1962 \\
\hline Land & Russland (XA-RU); USA (XD-US) \\
\hline Besetzung & $\begin{array}{l}\text { Sprecher, 2 } \\
\text { Sopran } \\
\text { Tenor <Stimmlage> } \\
\text { Bass <Stimmlage } ;, 2 \\
\text { Gemischter Chor (3-stimmig) } \\
\text { Orchester }\end{array}$ \\
\hline Wumerische Kennzeichnung & Werkverzeichnis-Zählung: K 98 \\
\hline Thematischer Bezug & Bibel. Genesis, 6,5-9,17 \\
\hline Systematik & $14.4 p$ Personen zu Musik \\
\hline Typ & Werk der Musik (wim) \\
\hline
\end{tabular}

Abb. 3: GND-Datensatz zu Igor Strawinsky: The flood, http://d-nb.info/gnd/300157800

\begin{tabular}{|c|c|}
\hline \multicolumn{2}{|l|}{$\overline{G N D}$} \\
\hline $\begin{array}{l}\text { Link zu diesem } \\
\text { Datensatz }\end{array}$ & http://d-nb.info/gnd/4399716-8 \\
\hline Komponist/Urheber & Strauss, Johann \\
\hline Titel des Werkes & An der schönen, blauen Donau \\
\hline Andere Titel & $\begin{array}{l}\text { Beautiful blue Danube } \\
\text { By the beautiful blue Danube } \\
\text { On the beautiful blue Danube } \\
\text { The blue Danube waltz } \\
\text { Le beau Danube } \\
\text { Donauwalzer } \\
\text { On the blue Danuve } \\
\text { Le beau Danube bleu } \\
\text { The blue Danube }\end{array}$ \\
\hline Quelle & JStraußWV \\
\hline Zeit & erstellt: 1867 \\
\hline Land & Österreich (XA-AT) \\
\hline Weitere Angaben & Besetzung: für Orchester mit Männerchor (TTBB) ad lib. \\
\hline Form & Walzer \\
\hline Besetzung & Orchester \\
\hline $\begin{array}{l}\text { Numerische } \\
\text { Kennzeichnung }\end{array}$ & Opus-Zählung: op. 314 \\
\hline $\begin{array}{l}\text { Thematischer } \\
\text { Bezug }\end{array}$ & Donau \\
\hline Systematik & 14.4p Personen zu Musik \\
\hline Typ & Werk der Musik (wim) \\
\hline
\end{tabular}

Abb. 4: GND-Datensatz zu Johann Strauss: An der schönen, blauen Donau, http://d-nb.info/gnd/4399716-8 
so das Verhältnis von Werken, Expressionen, Manifestationen und Exemplaren - im bibliothekarischen wie im wissenschaftlichen Kontext immer wieder neu zu diskutieren. Dabei nehmen Begriff und Definition des „musikalischen Werkes“ und seiner möglichen Ausprägungen eine zentrale Rolle ein. Denn unabhängig davon, dass Normierung immer auch Reduktion bedeutet, muss bedacht werden, dass das Modell der GND-Werknormdatei einem speziellen Werkbegriff verpflichtet ist. Es basiert auf der Vorstellung eines Grundtypus', auf den sich die abgeleiteten Instanzen (wie die Platon'schen Abbilder auf die Idee) beziehen. Was allerdings bei der Zusammenführung von verschiedenen Benennungen für dieselbe Sache gut funktioniert, weil in der Tat verschiedene Signifikanten immer auf dasselbe Signifikat rekurrieren, muss an seine Grenzen geraten, sobald ein komplexerer Umstand wie ein ,Werk ${ }^{c}$ ins Spiel kommt, dessen Begriffe und Konzepte höchstgradig historisch-kulturell bedingt sind und entsprechend kontextualisiert werden müssen. Ein Großteil der überlieferten Musik lässt sich nur schlecht mit einem Werkbegriff vereinbaren, der von einem abgeschlossenen, opus perfectum et absolutum' im neuzeitlichen Sinn mit benennbaren Kennzeichen wie Tonart, Gattung, Besetzung etc. ausgeht, auf den aber die GND für musikalische Werke setzt. Daneben könnten musikalische ,Werke' ebenso gut als Rhizom beschrieben werden, als vielgestaltiges Netz von Verbindungen, das sich zwar an einzelnen Stellen ,materialisiert‘ aber grundsätzlich unendliche alternative Möglichkeiten nicht nur der Realisierung, sondern auch der ontologisch übergeordneten ,Idee' bereithält und sich nicht auf einen einzigen Ursprung oder Zentralpunkt zurückführen lässt. ${ }^{13}$

Man kann und muss für das Konzept einer musikalischen Werk-Normdatei also grundlegend fragen: Was ist ,das Werk'? Wo endet es gegebenenfalls und beginnt ein neues? Wo haben musikalische „Werke“, die Zufälle der Aufführungssituation in ihr Konzept integrieren wie beispielsweise in der Neuen Musik, wo Jam-Sessions, wo Opern, die je nach Kontext verschiedene Arien zusammenstellen, wo verschiedene Fassungen einer Komposition ihre definierten Grenzen bzw. ihre eineindeutige Gestalt, die im Werk-Normdatensatz gefasst werden kann?

In der Begegnung mit diesen Phänomenen ist die $\mathrm{Ab}$ grenzung der FRBR-Ebenen Werk und Expression eine immer neue Herausforderung, für den bibliothekarischen

13 Zum Diskurs über Begriff und Definition musikalischer Werke s. stellvertretend Hinrichsen, Hans-Joachim: Musikwissenschaft und musikalisches Kunstwerk. Zum schwierigen Gegenstand der Musikgeschichtsschreibung. In: Lütteken, Laurenz (Hrsg.): Musikwissenschaft. Eine Positionsbestimmung. Kassel u. a. 2007, 67-87.
Alltag, aber ebenso und vielleicht noch zentraler für die wissenschaftliche (Nach-)Nutzung der Daten. Wie unklar zum Beispiel der Umgang mit Werkfassungen in der GND ist, zeigt sich an zwei so zentralen Kompositionen wie Johann Sebastian Bachs Matthäuspassion (BWV 244/244 a-b) und seiner Johannespassion (BWV 245, 245 a-c), deren jeweilige Fassungen auf verschiedene Weisen in der GND repräsentiert werden. Für die Matthäuspassion BWV 244 (b) führt die GND zwei Werkdatensätze (,wim“), die miteinander unspezifisch (als „Bezug zu Werken“) verlinkt sind, wobei der Datensatz zu BWV 244b „Frühfassung“ im Titel enthält (Abb. 5 und 6). Beide Fassungen sind damit als gleichberechtigte Werke in der GND abgelegt.

Die vier verschiedenen Versionen der Johannespassion BWV 245(a-c) jedoch vereint die GND in einem einzigen Datensatz (,wim“ Abb. 7), die alternativen Fassungen werden in einem nicht normierten und verknüpften Freitext-Kommentarfeld (,weitere Angaben“) untergebracht. Auch die Angaben zur Unterteilung der Werkverzeichnisnummern fehlen. Eine Differenzierung geschieht erst auf der Ebene der Manifestationen (z. B. der Titeldaten im Bibliothekskatalog), sie ist in der Normdatei also nicht mehr sichtbar und nicht mehr wirksam.

Unter der Voraussetzung, dass die Informationen der GND den aktuellen wissenschaftlichen Konsens widerspiegeln soll - wofür die Quellenangaben sprechen -, müssten konsequenterweise eigene Werkverzeichnisnummern auch zu eigenständigen Datensätzen führen. Daran anschließend ließe sich überlegen, inwiefern Fassungen und Bearbeitungen im FRBR-Sinne selbst Werke oder eben Expressionen des Werkes darstellen und auch auf dieser Ebene (als ,wie“-Satz) in der GND abgebildet werden sollten. In zweitem Falle bliebe die Werk-Ebene ein Abstraktum, auf das alle Fassungen (als jeweils eigene Expressionen) rekurrieren. So verlockend dieses Modell sein mag: Es auf alle Kompositionen auszurollen - unabhängig davon, ob von ihnen bereits verschiedene Fassungen bekannt sind oder nicht -, dürfte weder in der bibliothekarischen noch in der wissenschaftlichen Community vermittelbar sein. Trotzdem könnte die Möglichkeit zur für die Musik so wichtigen Unterscheidung von Werk und Expression stärker genutzt werden, um die Zusammenhänge von kulturellem Erbe und historischen Ausprägungen stärker zu verdeutlichen. Sieht man von 5000 kürzlich angelegten „wie“-Sätzen für historische Tonaufnahmen von musikalischen Werken ab (als „aufgeführte Musik“ mit dem zu Grunde liegenden Werktitel verknüpft), ${ }^{14}$ die in einem

14 Vgl. z.B. http://d-nb.info/gnd/107137821X Als Merkmale, vgl. IFLA (Anm. 6) 21, wurden hier neben der Art der Expression (aufge- 


\begin{tabular}{|c|c|}
\hline \multicolumn{2}{|l|}{$\overline{\mathrm{ANO}}$} \\
\hline $\begin{array}{l}\text { Link zu diesem } \\
\text { Datensatz }\end{array}$ & http://d-nb.info/gnd/300009798 \\
\hline Komponist/Urheber & Bach, Johann Sebastian \\
\hline Titel des Werkes & Matthäuspassion \\
\hline Beteiligt & Verfasser / Urheber: Henrici, Christian Friedrich \\
\hline Andere Titel & $\begin{array}{l}\text { Matthäuspassion, BWV } 244 \\
\text { Saint Matthew passion } \\
\text { Passion selon Saint Matthieu } \\
\text { St. Matthew passion } \\
\text { Matthäus Passion } \\
\text { Matthäus-Passion } \\
\text { Passio de D.N.J.C. secundum Matthaeum } \\
\text { Passion unseres Herrn Jesu Christi nach dem Evangelisten Matthäus } \\
\text { Passion of our Lord Jesus Christ according to St. Matthew } \\
\text { Passions-Musik nach dem Evangelisten Matthäus } \\
\text { Passionsmusik nach dem Evangelisten Matthaeus } \\
\text { Passio Domini nostri Jesu Christi secundum evangelistam Matthaeum }\end{array}$ \\
\hline Quelle & BWV \\
\hline Zeit & erschienen: 1736 \\
\hline Land & Deutschland (XA-DE) \\
\hline Sprache(n) & Deutsch (ger) \\
\hline Form & Passionsmusik \\
\hline Besetzung & $\begin{array}{l}\text { Sopran, } 2 \\
\text { Alt < Stimmlage>, } 2 \\
\text { Tenor <Stimmlage>, } 2 \\
\text { Bass <Stimmlage>, } 2 \\
\text { Gemischter Chor, } 2 \text { (8-stimmig (SATB, SATB)) } \\
\text { Orchester, } 2\end{array}$ \\
\hline $\begin{array}{l}\text { Numerische } \\
\text { Kennzeichnung }\end{array}$ & $\begin{array}{l}\text { Werkverzeichnis-Zählung: BWV } 244 \\
\text { Werkverzeichnis-Zählung: BC D } 3 \text { b }\end{array}$ \\
\hline Bezug zu Werken & Bach, Johann Sebastian: Matthäuspassion. Frühfassung \\
\hline DDC-Notation & 782.23 \\
\hline Systematik & 14.4p Personen zu Musik \\
\hline Typ & Werk der Musik (wim) \\
\hline
\end{tabular}

Abb. 5: GND-Datensatz zu Johann Sebastian Bach: Matthäuspassion, http://d-nb.info/gnd/300009798

\begin{tabular}{|c|c|}
\hline \multicolumn{2}{|l|}{$\overline{\overline{\mathrm{ANO}}}$} \\
\hline $\begin{array}{l}\text { Link zu diesem } \\
\text { Datensatz }\end{array}$ & http://d-nb.info/gnd/4634416-0 \\
\hline Komponist/Urheber & Bach, Johann Sebastian \\
\hline Titel des Werkes & Matthäuspassion. Frühfassung (Maschinelle Umsetzung GND) \\
\hline Andere Titel & $\begin{array}{l}\text { Frühfassung der Matthäuspassion (RDA) } \\
\text { Matthäuspassion, BWV 244b }\end{array}$ \\
\hline Quelle & BWV \\
\hline Land & Deutschland (XA-DE) \\
\hline Sprache(n) & Deutsch (ger) \\
\hline Weitere Angaben & Frühfassung in der Abschrift von Johann Christoph Altnickol \\
\hline Form & Passionsmusik \\
\hline $\begin{array}{l}\text { Numerische } \\
\text { Kennzeichnung }\end{array}$ & $\begin{array}{l}\text { Werkverzeichnis-Zählung: BWV 244b } \\
\text { Werkverzeichnis-Zählung: BC D } 3 a\end{array}$ \\
\hline Bezug zu Werken & Bach, Johann Sebastian: Matthäuspassion \\
\hline Systematik & 14.4p Personen zu Musik \\
\hline Typ & Werk der Musik (wim) \\
\hline
\end{tabular}

Abb. 6: GND-Datensatz zu Johann Sebastian Bach: Matthäuspassion - Frühfassung, http://d-nb.info/gnd/4634416-0 


\begin{tabular}{|c|c|}
\hline \multicolumn{2}{|l|}{$\overline{\underline{a n 0}}$} \\
\hline $\begin{array}{l}\text { Link zu diesem } \\
\text { Datensatz }\end{array}$ & http://d-nb.info/gnd/300006667 \\
\hline Komponist/Urheber & Bach, Johann Sebastian \\
\hline Titel des Werkes & Johannespassion \\
\hline Andere Titel & $\begin{array}{l}\text { Johannespassion, BWV } 245 \\
\text { Johannes-Passion } \\
\text { St. John passion } \\
\text { Johannes Passion } \\
\text { Passion selon Saint Jean } \\
\text { Passio secundum Joannem } \\
\text { Grosse Passions-Musik nach dem Evangelisten Johannes } \\
\text { Passionsmusik nach dem Evangelisten Johannes } \\
\text { Passion Jesu Christi nach dem Evangelisten Johannes }\end{array}$ \\
\hline Quelle & BWV \\
\hline Zeit & erschienen: 1724 \\
\hline Land & Deutschland (XA-DE) \\
\hline Sprache(n) & Deutsch (ger) \\
\hline Weitere Angaben & $\begin{array}{l}\text { Datum der ersten Aufführung } 1724 \\
\text { Vier verschiedene Versionen: } 1.1724 ; 2.1725 ; 3.1728 \text { oder } 1730 ; 4 . \\
\text { frühestens } 1746 \text { und spätestens } 1749 \\
\text { BWV } 245 \text { ist die maßgebliche Werkverzeichniszählung }\end{array}$ \\
\hline Form & Passionsoratorium \\
\hline Besetzung & $\begin{array}{l}\text { Sopran } \\
\text { Alt <Stimmlage> } \\
\text { Tenor <Stimmlage> } \\
\text { Bass <Stimmlage> } \\
\text { Gemischter Chor (4-stimmig (SATB)) } \\
\text { Querflöte, } 2 \\
\text { Oboe, } 2 \\
\text { Violine, } 2 \\
\text { Viola d'amore, } 2 \\
\text { Viola } \\
\text { Viola da gamba } \\
\text { Laute } \\
\text { Basso continuo }\end{array}$ \\
\hline $\begin{array}{l}\text { Numerische } \\
\text { Kennzeichnung }\end{array}$ & $\begin{array}{l}\text { Werkverzeichnis-Zählung: BWV } 245 \\
\text { Werkverzeichnis-Zählung: BC D } 2 \text { a } \\
\text { Werkverzeichnis-Zählung: BC D } 2 \text { b } \\
\text { Werkverzeichnis-Zählung: BC D } 2 \text { c } \\
\text { Werkverzeichnis-Zählung: BC D } 2 \text { d } \\
\text { Werkverzeichnis-Zählung: BC D } 2 \text { e }\end{array}$ \\
\hline Systematik & 14.4p Personen zu Musik \\
\hline Typ & Werk der Musik (wim) \\
\hline
\end{tabular}

Abb. 7: GND-Datensatz zu Johann Sebastian Bach: Johannespassion, http://d-nb.info/gnd/300006667

kürzlich abgeschlossenen Drittmittelprojekt der SLUB Dresden entstanden, ${ }^{15}$ bleiben in der GND nur etwa 70 „wie“-Sätze, die sich alle - außerhalb des musikwissen-

führte Musik), das Aufnahmedatum (date of expression), die Ausführenden mit ihren Funktionen (medium of performance of expression) und als weitere disambiguierende Information der Ort der Aufnahme erfasst.

15 https://www.slub-dresden.de/ueber-uns/projekte/juengst-abges chlossene-projekte/standardisierung-erschliessungsdaten-tontrae ger/. Vgl. Grzondziel, J.; Neuendorf, S.; Scheidler, K.: Eine diskografische Normdatei - Datensätze für historische Aufnahmen in der Gemeinsamen Normdatei. In: Forum Musikbibliothek 36 (3) (2015), 43-46. schaftlichen Kontextes - auf Übersetzungen von Werken der Literatur beziehen.

Die bisher noch bestehenden strukturellen Unklarheiten zwischen den FRBR-Ebenen und FRAD-Anforderungen bei musikalischen Werken und Expressionen ${ }^{16}$ erschweren eine effektive Nachnutzung der GND-Daten momentan zwar noch, bei einem wachsenden Datenpool und weiter reichenden Nutzungsszenarien sollten aber Konzept und Umsetzung musikalischer Werknormdaten in der GND im

16 So werden beispielsweise Expressions- und Manifestationskategorien wie die Sprache des Werkes oder Aufführungs- und Veröffentlichungsdaten in den Werksatz aufgenommen. 


\begin{tabular}{|c|c|}
\hline \multicolumn{2}{|l|}{$\overline{\underline{Q N 0}}$} \\
\hline $\begin{array}{l}\text { Link zu diesem } \\
\text { Datensatz }\end{array}$ & http://d-nb.info/gnd/110137893X \\
\hline Komponist/Urheber & Verdi, Giuseppe \\
\hline Titel des Werkes & Rigoletto (aufgeführte Musik), xxB 87242 \\
\hline Beteiligt & $\begin{array}{l}\text { Librettist: Piave, Francesco Maria ; Sänger: Ebers, Clara ; Sänger: Sabel, } \\
\text { Jakob ; Sänger: Wocke, Hans ; Grüber, Arthur(Dirigent) ; Sänger: Deutsches } \\
\text { Opernhaus Berlin. Chor ; Instrumentalmusiker: Deutsches Opernhaus Berlin. } \\
\text { Orchester ; Hersteller: Carl-Lindström-Aktiengesellschaft (Berlin) }\end{array}$ \\
\hline Andere Titel & Rigoletto (aufgeführte Musik), P xxB 8724-2 \\
\hline Quelle & $\begin{array}{l}\text { Zwarg, Christian [Hrsg.]: Beka Discography: } \\
\text { http://discography.phonomuseum.at/odeon/odmxB.pdf }\end{array}$ \\
\hline Zeit & erstellt: 07.01 .1940 \\
\hline Geografischer Bezug & Herstellungsort: Berlin \\
\hline Weitere Angaben & $\begin{array}{l}\text { Titel bei Zwarg: Rigoletto : Querschnitt, Teil } 1 \\
\text { Aufnahme: Carl Lindström AG, SO.33, Schlesische Straße 26, Berlin }\end{array}$ \\
\hline Besetzung & $\begin{array}{l}\text { Singstimme, } 3 \\
\text { Chor } \\
\text { Orchester }\end{array}$ \\
\hline Oberbegriffe & Beispiel für: Schallaufzeichnung \\
\hline Bezug zu Werken & Verdi, Giuseppe: Rigoletto \\
\hline Systematik & 14.1 Musik (Allgemeines), Musikgeschichte \\
\hline Typ & Expression (wie) \\
\hline
\end{tabular}

Abb. 8: GND-Datensatz zu Guiseppe Verdi: Rigoletto (aufgeführte Musik), http://d-nb.info/gnd/110137893X

engen Austausch zwischen Bibliothek und Wissenschaft weiter diskutiert und geschärft werden (vgl. auch weiter unten).

\section{Potenzial musikalischer Werk-Normdaten}

In bibliothekarischen, aber auch in wissenschaftlichen Kontexten werden Normdaten seit Langem zur ökonomischen Wissensorganisation eingesetzt. Diese Szenarien beschränken sich bisher allerdings zumeist auf die über Verlinkung realisierbare Verweisungsfunktion in einem identifizierenden und zuordnenden Vorgang, bei dem die ID des Normdatums stellvertretend für die identifizierte Entität steht. ${ }^{17}$ Über ihre Funktion als Spezifikation eines (Personen-, Orts- oder Werk-)Namens hinaus werden die für die Disambiguierung notwendigen kontextualisierenden Inhalte der GND trotz umfangreicher Aktivitäten der

17 Z.B. in digitalen Editionsprojekten, in der Wikipedia, die über BEACONs (einem von der DNB entwickelten Format zur Verlinkung auf die GND) ihre Informationen mit den passenden GND-Einträgen verknüpft oder in Projekten wie „Bilder der Revolte“, für das die GND explizit als Datenlieferant im Rahmen eines Kulturhackathons auftrat, https://codingdavinci.de/daten und http://bilder-der-revolte.d e/ und http://bilder-der-revolte.de/coding-da-vinci.
Deutschen Nationalbibliothek ${ }^{18}$ derzeit jedoch noch kaum systematisch zur Verknüpfung vorhandenen und damit zur Generierung neuen Wissens genutzt.

Bedenkt man, dass (musikalische) Werksätze erst 2012 mit der GND eingeführt wurden, ${ }^{19}$ verwundert es nicht,

18 Die DNB beteiligt seit mehreren Jahren an der Entwicklung von Konzepten und der Bereitstellung technischer Schnittstellen und vergrößert so den Pool an Daten- und Beziehungsmaterial im semantischen Netz (Generell stehen alle GND-Daten unter einer CCO Lizenz frei einer Nachnutzung zur Verfügung. Vgl. auch Haffner, Alexander: Internationalisierung der GND durch das Semantic Web. Frankfurt am Main 2012). Normdaten sind dabei nicht nur ein beliebiges Element neben anderen, sie erfüllen vielmehr eine Funktion als „Rückgrat eines maschinenlesbaren, semantischen Netzes der Kultur und Wissenschaft“ (Kett, Jürgen: GND Entwicklungsprogramm 2017-2021. Frankfurt am Main 2017, 2). Verschiedene Projekte beschäftigen sich außerdem mit der Frage, wie „open Data“ tatsächlich zu „linked“ Data über System- oder Projektgrenzen hinweg werden und neue Erkenntnisse ermöglichen (vgl. z. B. Culturegraph http://www.culture graph.org/Subsites/culturegraph/DE/Home/home_node.html, wikidata (https://de.wikipedia.org/wiki/Wikidata, dazu auch Voß, Jakob et al.: Normdaten in Wikidata. Hannover 2014) oder dbpedia (https://de.wikipedia.org/wiki/DBpedia)). Von Seiten der DNB wurde eine verbesserte Sicht- und Nutzbarkeit der GND-Daten als erklärtes Ziel innerhalb ihrer strategischen Prioritäten für die nächsten Jahre formuliert (DNB: Strategische Prioritäten. Frankfurt 2017, 14f.).

19 Als Einheitssachtitel des DMA wurden zuvor nur alternative Benennungen für musikalische Werke geführt, Werknormdaten für Werke der Literatur und bildenden Kunst wurden ebenfalls erst mit der GND eingeführt. 
dass deren Nutzung noch hinter der Verwendung anderer Normdatentypen (wie der Personennormdatei) zurücksteht. Selbst im bibliothekarischen Kontext entfalten sie ihre Wirksamkeit noch nicht ganz, was vermutlich als Übergangsphänomen zu werten und auf den hohen Spezialisierungsgrad des Entitätentyps zurückzuführen ist. Der primäre Nutzen des Normsatzes im bibliothekarischen Umfeld liegt darin, alle individuellen Ausprägungen eines Werkes über einen Identifikator zusammenzufassen und als valides Suchergebnis im Bibliothekskatalog alle Manifestationen und Exemplare des Werkes anzuzeigen. Die im Normsatz aufgeführten Titelvarianten erlauben zudem konsistente Ergebnisse bei unterschiedlichen Suchtermini. Es ist der bisher nur geringen Anzahl der mit Werknormdaten verknüpften bibliografischen Daten und fehlender technischer Aufrüstungen geschuldet, dass diese Grundfunktionalitäten allerdings nur für eine Teilmenge der Daten und nur in ausgewählten Bibliothekskatalogen und Discoverysystemen wirksam werden. Bei einer Suche im Online-Katalog des Südwestdeutschen Bibliotheksverbunds (SWB) nach Modest Musorgskijs Sinfonischer Dichtung „Eine Nacht auf dem kahlen Berge“ beispielsweise erhält man unabhängig von den Suchtermini - wie „Ivanova noč' na lysoj gore“ oder „Night on the bare mountain“ oder „Sint-Jansnacht op de kale berg“ etc. - mindestens immer dieselben 34 Titeltreffer, die mit dem Normdatensatz verbunden sind und zu denen damit 13 Titelvarianten indexiert sind (Abb. 9). Ergänzend erscheinen bei den verschiedenen Suchtermini viele weitere Treffer, die in ihren bibliografischen Daten ohne Normdatenverknüpfung unterschiedliche Titelvarianten mitführen. Wirklich konsistente Ergebnisse ließen sich nur durch eine retrospektive Normdatenverknüpfung erreichen.

Aber nicht nur der Vorteil der schnellen Identifizierung und Disambiguierung über die GND-Verlinkung wird mit den Normdatensätzen noch nicht vollumfänglich im OPAC fruchtbar. Bisher werden auch die kontextualisierenden Informationen der GND-Datensätze nicht indexiert und damit nicht recherchierbar gemacht. Dabei erlaubte ein Abrufen der in den Werknormdaten hinterlegten Informationen über Gattungszugehörigkeit, Besetzung, chronotopische Beziehungen für den Bibliothekskatalog neue Such- und Filtermöglichkeiten, die bisher höchstens durch zusätzlich aufgenommene Daten der Titelaufnahme bereitstehen. Anlässlich einer Vernissage mit Bildern finnischer Gegenwartskunst will ich ein musikalisches Programm mit Stücken für Sopran und Klavier finnischer Komponisten der Jahrtausendwende zusammenstellen? Die Informationen der musikalischen Werknormdatei müssten nur mit den Titel- und Exemplardaten meines Bibliothekskataloges verbunden und entsprechend inde- xiert werden, um mir Auskunft über ein passendes Repertoire $\mathrm{zu}$ geben..$^{20}$ Bis dato ist die zeitliche und geografische Einschränkung in den meisten OPACs nicht möglich, ein Filtern der Besetzung erfolgt nur, wenn zusätzlich zur Formalerschließung der Manifestationen eine Sacherschließung nach Form und Gattung vorgenommen wird, wie dies zum Beispiel die Bibliotheken des Südwestdeutschen Bibliotheksverbunds mit großer Konsequenz betreiben. Die Nutzung der in den Werknormdatensätzen enthaltenen Informationen für den Bibliothekskatalog hätte also zwei Effekte: Sie verbesserte die Recherche mit neuen Sucheinstiegen und würde es erlauben, die lokale Sacherschließungsarbeit auf die Fälle zu reduzieren, in denen Informationen wie Besetzung oder Gattung der Manifestationen von denen der Werke abweichen. Eine zentrale und kollaborative Pflege der Werksätze in der GND dürfte deshalb zu größerer Arbeitseffizienz aber auch zu einer stärkeren Qualitäts- und Datensicherung beitragen. Setzen diese Szenarien auf eine konsequentere Nutzung der GND-Informationen in den Erfassungs- und Suchsystemen der Bibliothek(sverbünde) selbst, sind sie genauso im Umgang mit externen Datenquellen bei der Gestaltung von Discoverysystemen mitzudenken. ${ }^{21}$

Als Projekt auf der Grenze zwischen bibliografischem Nachweisinstrument und digitaler Musikwissenschaft erweitert das Internationale Quellenlexikon der Musik (RISM) derzeit sein Datenformat um einen Werknormsatz, der

20 Einen ersten Ansatz bietet das Vufind-basierte Discovery-System der Hochschule für Musik und Theater „Felix Mendelssohn Bartholdy“ Leipzig (https://katalog.hmt-leipzig.de/). Vgl. auch Hofmann, Anke; Wiermann, Barbara: The VuFind based MT-Katalog - a customized music library service at the University of Music and Drama Leipzig. In: Lausen, Berthold; Krolak-Schwerdt, Sabine; Böhmer, Matthias (Hrsg.): Data Science, Learning by Latent Structures, and Knowledge Discovery. Berlin 2015, 547-56 und Hofmann, Anke; Wiermann, Barbara: Customizing music discovery services - Experiences at the Hochschule für Musik und Theater Leipzig. In: Music Reference Service Quarterly 17 (2014), 61-75. Auch an der Bibliothek der Universität für Musik und darstellende Kunst Wien wird die Nutzung der Werknormdateninformationen im Discovery-System momentan getestet, vgl. Christoph Steiger: Präsentation des Notenbestandes mithilfe der GND, Vortrag auf der Tagung der AIBM, Münster 2017, http://www.aibm.info/wp-co ntent/uploads/2017/09/Steiger_gnd-primo-mit-notizen.pdf.

21 So erweiterte der größte Anbieter freier Notendigitalisate, das International Music Score Library Project, vor einigen Jahren seine Metadaten um VIAF-Nummern zu musikalischen Werken. In verschiedenen Discovery-Systemen werden die IMSLP-Inhalte zwar eingebunden, die Normdateninformationen aber nicht ausgewertet und weiter verarbeitet und so auf eine Verbesserung der Metadatenqualität und damit der Recherchierbarkeit der Materialien verzichtet. Daten aus IMSLP finden sich derzeit in den Discoverysystemen des sächsischen Vufind-Konsortiums und in der von der Bayerischen Staatsbibliothek betriebenen Vifa-Musik-Suche. 


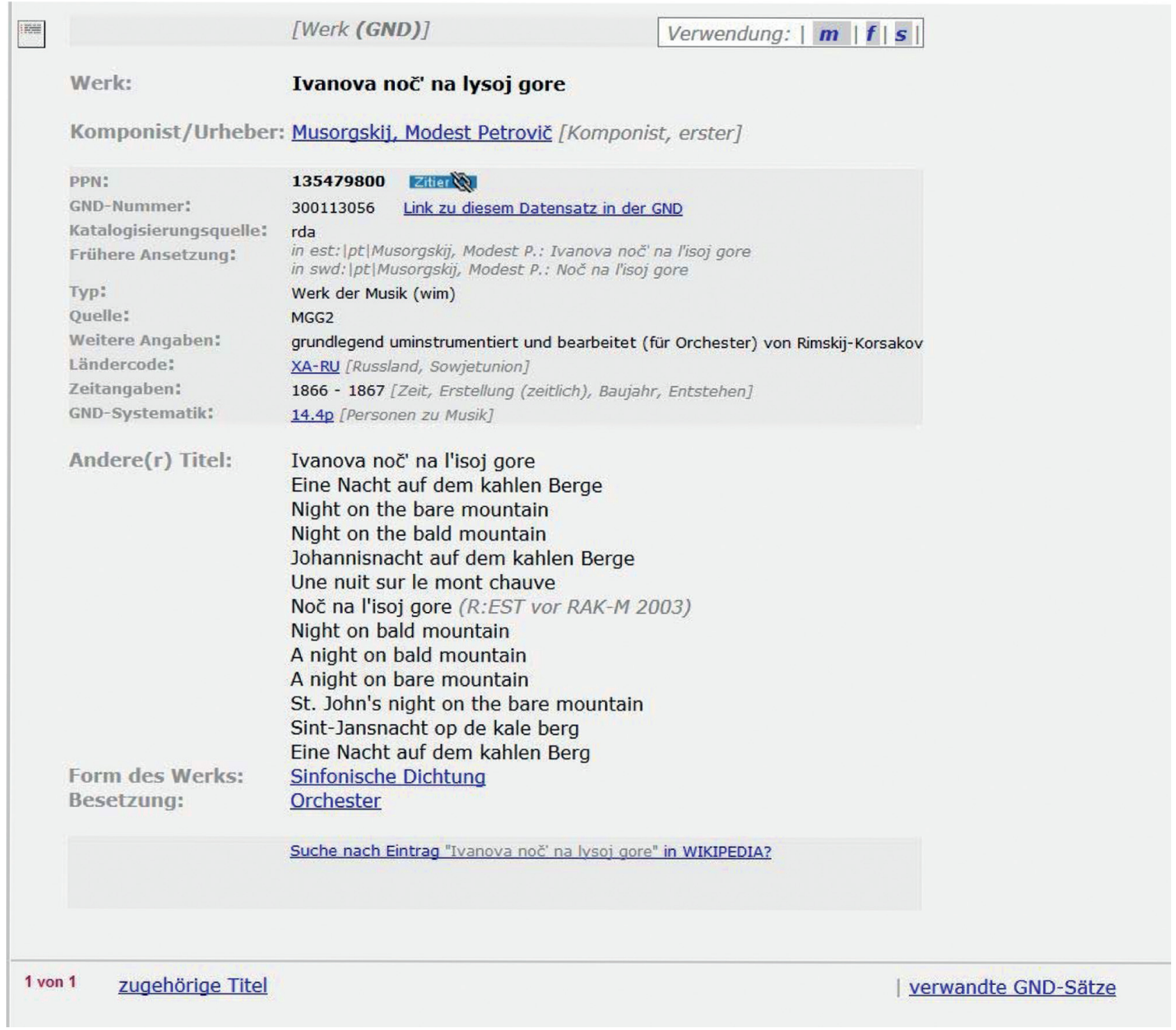

Abb. 9: Südwestdeutscher Bibliothekskatalog. Normdatensatz für M. Musorgskij: Eine Nacht auf dem kahlen Berge, http://swb.bsz-bw.de/DB=2.1/PPNSET?PPN=135479800\&INDEXSET=1

VIAF- und GND-Nummer mitführt. ${ }^{22}$ Vor dem Hintergrund, dass nirgends die Varianz eines Werkes so deutlich wird, wie in einem Quellenlexikon, dessen verzeichnete Handschriften eine große Vielfalt abbilden, bietet das Projekt optimale Möglichkeiten, die Kompatibilität zwischen einem Normierungswunsch und wissenschaftlich notwendiger Differenzierung auszutesten und den Werkbegriff umfänglich zu reflektieren. Bei der Einbindung von Werknormsätzen wird man im Kontext von RISM zudem mit einer hohen Anzahl an unikalen Werken konfrontiert, die bis dato in keiner Normdatenbank erfasst sind und zu de-

22 Vgl. den Artikel „Das Internationale Quellenlexikon der Musik, RISM“ von Keil und Pugin in diesem Heft S. 309-318. nen häufig kaum über die Quelle hinausgehende Informationen vorliegen, was das Konzept eines Normdatensatzes leicht in Frage stellen kann. Eine besondere Herausforderung liegt des Weiteren im internationalen Anspruch des Projekts, für das die VIAF-Daten Vorrang haben vor der GND. Dies bedeutet praktisch, dass die Datenlage nochmals heterogener und das Neuanlegen von Normsätzen mit einem mehrstufigen Prozess verbunden ist. RISM, als internationales Spezialprojekt, lässt derzeit eine Direktanbindung an VIAF prüfen, wie sie wenige andere Spezialprojekte bereits unterhalten. Für RISM bedeutet die Einführung von Werknormdaten nicht nur innerhalb der Datenbank selbst eine Verbesserung der Darstellung von verschiedenen zu einem Werk gehörigen Quellen, wobei es sich bei diesen Manifestationen um Skizzen, Autografe, Abschrif- 
ten und Drucke handeln kann. Sie erhöht zudem deutlich die Anschlussfähigkeit des Produkts und wertet die bibliografischen Daten als Forschungsdaten nachhaltig auf. ${ }^{23}$

Unter der Perspektive ,Forschungsdaten' können die musikalischen Werknormsätze zudem ein bis dato fast ungenutztes Potenzial entfalten, welches anhand dreier Projekt(ideen) exemplarisch dargestellt werden soll.

Im Rahmen des DFG-geförderten Fachinformationsdienstes Musikwissenschaft entsteht derzeit an der SLUB Dresden das Nachweissystem „musiconn.performance“ für Konzerte, Opernaufführungen, Musicaldarbietungen und jedwede Art von musikalischen Veranstaltungen. ${ }^{24}$ Dabei werden Informationen zu Daten, Orten, aufgeführten Werken, beteiligten Personen etc. aus verschiedenen Quellen wie Ankündigungen, Programmheften oder Rezensionen abgelegt. Die Verknüpfung der Entitäten mit den jeweiligen Normdaten der GND erlauben es, Kontextinformationen zuzuspielen, die in der Programmdatenbank nicht noch einmal aufgenommen und gepflegt werden müssen. Natürlich können sich dabei Widersprüche ergeben: Ein Sänger wird im zugehörigen Normdatum als Tenor geführt, ist beim Konzert aber als Bass nachgewiesen oder ein Werk ist im Normdatum mit Violine und Klavier besetzt angegeben, wird aber mit Flöte und Gitarre aufgeführt. Derartige Widersprüche müssen - sofern sie sich nicht als so allgemein erweisen, dass eine Korrektur oder Ergänzung des Normdatensatzes sinnvoll ist - zugelassen werden und sichtbar bleiben - sie bieten als projektspezifische Information wichtige Auskünfte über konkrete Ausprägungen des Normdatums. Die Ausführung des Werkes mit Flöte und Gitarre statt Violine und Klavier wäre dann eine Expression des zugrunde liegenden Werkes. ${ }^{25}$ Nichtsdestotrotz ließe eine Kombination der aufgenommenen Daten mit den Normdaten der GND Fragen zu wie: Gab es an bestimmten Orten zu bestimmten Zeiten eine Vorliebe für Aufführungen bestimmter Gattungen? Bestimmter Besetzungen? Musik bestimmter Sprachen? Oder Musik von Komponisten bestimmter Herkunft? Diesbezügliche Informationen kämen direkt aus der Normdatei, würden ggf. ergänzt durch kontrastierende Aussagen aus dem Quellenmaterial, qualifizieren die Daten-

23 Eine erste Auswertung von RISM-Daten als Forschungsdaten wurde von Stephen Rose, Sandra Tuppen und Loukia Drosopoulou vorgenommen. Vgl. dieselb.: Writing a Big Data history of music. In: Early Music 43 (4) (2015), 649-60, doi: 10.1093/em/cav071.

24 musiconn.performance ist als dezentral nutzbares Werkzeug angelegt, das von verschiedenen Wissenschaftlern zur Datenerfassung und Dokumentation verwendet werden kann. Ebenso sind bereits erhobene Datenbestände integrierbar.

25 Inwiefern für diese Expression ein eigener Expressionsnormdatensatz angelegt wird, ist eine weitergehende Frage. bank aber in jedem Fall um Informationen, die zentral gepflegt - d.h. dem Forschungsstand entsprechend erweitert und korrigiert - und ohne eigenen Aufwand den eigenen Daten hinzugegeben werden können.

Eine andere Perspektive auf musikalisches Leben wählt eine Projektidee, die sich der Programmpolitik Leipziger Musikalienverleger des 19. und frühen 20. Jahrhunderts zuwendet. Ausgehend von der Prämisse, dass Verlagsprogramme (auch) aus ökonomischen Überlegungen zu Nachfrage und Absatzmöglichkeiten zusammengestellt werden, macht das Projekt Quellenmaterial zugänglich, indem Wirtschaftsbücher dreier Verlage mit den entsprechenden Zahlen digitalisiert, vor allem aber in eine Datenbank überführt werden. Dieses Material enthält Angaben zu Produktionsund Verkaufsmengen der verlegten Musikalien. Auch hier gilt: Werden die in den Musikdrucken enthaltenen Werke mit ihrem in der GND hinterlegten Normdatum verknüpft und die dort enthaltenen Kontextinformationen zugespielt, lassen sich sehr viel weiter reichende Fragen sehr viel schneller beantworten als es das Quellenmaterial auf den ersten Blick gestattet. Nun kann nicht nur abgelesen werden, welche konkreten Verlagsprodukte welche Konjunkturen erlebten, sondern wiederum gegengeprüft werden, ob es sich um Trends handelt, die bestimmte Gattungen, Besetzungen, Herkünfte etc. meinen. Und auch hier gilt, dass Widersprüche zwischen den Angaben im verlinkten Normdatum und konkreten Verlagsprodukten (als Manifestationen des Werkes) wiederum Hinweise auf historisch bedingte Besonderheiten geben, die nachzuverfolgen Teil der Forschungsfrage sein können.

Ein letztes Beispiel sei angefügt, auch wenn das Prinzip der Normdatennachnutzung dasselbe bleibt. In seinem Dissertationsprojekt zur Rezeption von (Erst-)Aufführungen von Guiseppe Verdis Requiem im deutschsprachigen Raum wertet Torsten Roeder 326 Rezensionen aus. ${ }^{26}$ Grundlage für die Dokumentenanalysen und seine Fragen nach bestimmten Beschreibungstopoi sind die semantisch ausgezeichneten und annotierten historischen Artikel, die als Forschungsdatenmaterial die Thesen der Arbeit empirisch stützen, gleichwohl aber auch zur Nachnutzung bereitstehen und einladen. ${ }^{27}$ Die Edition

26 Die Dissertation ist noch nicht veröffentlicht, einen Eindruck des Vorhabens vermittelt aber die Zusammenfassung zu Torsten Roeders Vortrag „Horizontales Lesen: Das ,Verdi-Requiem“ und die deutsche Kritik“ auf der Tagung der DhD in Köln, in: Vogeler, Georg (Hrsg.): DHd 2018 Kritik der digitalen Vernunft, Konferenzabstracts. Köln 2018, 232-35, dhd2018.uni-koeln.de/wp-content/uploads/boa-DHd20 18-web-ISBN.pdf [14.3.2018]

27 http://verdi-requiem.de (14.3.2018, Nutzername und Passwort: dhd2018). 


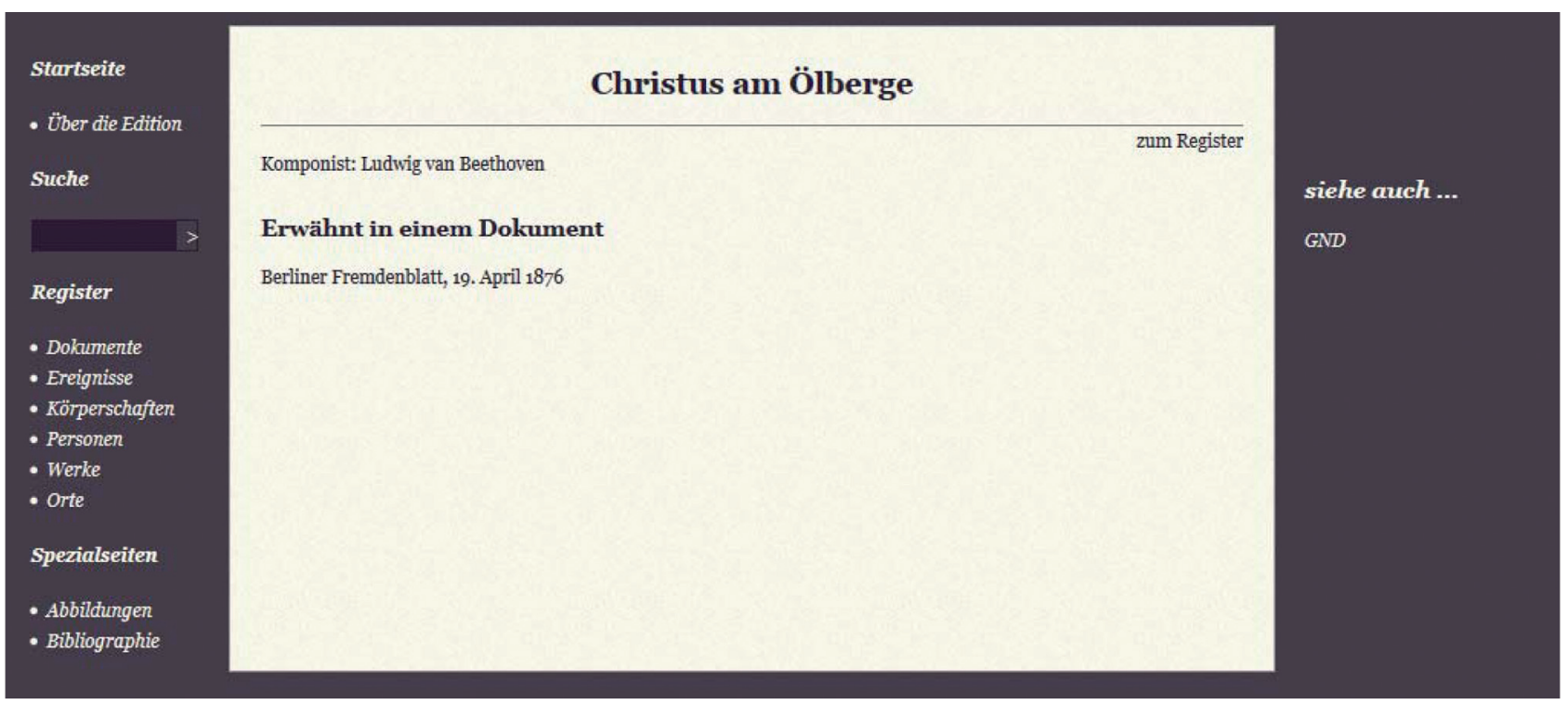

Abb. 10: Torsten Roeder: Werkregister zu verdi-requiem.de, http://verdi-requiem.de/opus?nym=beethoven.christus-am-oelberge

der Dokumente ist über verschiedene Register u.a. zu Personen, Orten und Werken erschließbar. Die dortigen Einträge sind über einen Verweis mit verschiedenen globalen Normdateien ${ }^{28}$ verlinkt.

Abgesehen von der Tatsache, dass zu etlichen Entitäten kein Normdateieintrag verfügbar ist, bietet sich konzeptionell auch hier an, die in den globalen Normdateien enthaltenen Informationen für Analysen zu nutzen: Wurde eine Verdi-Aufführung besonders häufig in Kombination mit anderen Kompositionen italienischer Provenienz besprochen? Wurde sie besonders häufig mit Kompositionen anderer Gattungen verglichen?

Dass sich diese drei Projekte über die Normdatenverknüpfung zudem gegenseitig erhellen, braucht nicht betont zu werden: Die Konzertprogrammdatenbank könnte Auskunft darüber geben, ob in zeitlicher oder räumlicher Nachbarschaft der besprochenen Verdi-Requiem-Aufführung italienische Opern aufgeführt wurden, das Musikverlagsprojekt darüber, welche Gattungen in jenem Zeitraum besonders gut verkauft werden konnten. Die drei angedeuteten Anwendungsfälle beschreiben die Nachnutzung der GND-Daten für jeweils spezifische Fragen. Die dort (in der Konzertprogrammdatenbank, in der Rezensionensammlung, im Forschungsprojekt zur Verlagsproduktion) erhobenen projektspezifischen Informationen fließen dabei zwar nicht direkt in die GND zurück, indirekt werden sie aber sehr wohl fruchtbar. Durch die Verlinkung der projekt-

28 VIAF, GND, ggf. Lexikoneinträge und Thesauri. Hier macht sich der Umstand, dass Normdatensätze für musikalische Werke (noch) nicht von externen Forschern angelegt werden können, besonders schmerzlich bemerkbar. spezifischen Entitäten mit den Datensätzen der GND wird die GND zum Scharnier, das die Informationen der verschiedenen Projekte wiederum miteinander kombinierbar macht.

Fruchtbar werden die musikwissenschaftlichen Erkenntnisse für die GND aber auch dadurch, dass die Diskussion von Konzept und Modell musikalischer Werknormdaten bereichert wird. So hat beispielsweise das DFGgeförderte Projekt „Quellenkorpus Bach-Söhne“, das in erster Linie dem weiteren Ausbau der Plattform Bach-digital ${ }^{29}$ dient, es sich zur Aufgabe gemacht, zu den Kompositionen Johann Sebastian Bachs und seiner Söhne Wilhelm Friedemann, Carl Philipp Emanuel, Johann Christian und Johann Christoph Friedrich, die zugehörigen GND-Datensätze anzulegen, zu ergänzen, zu bereinigen und schließlich mit den Informationen der Projektdatenbank zu verknüpfen. Im Rahmen des Projektes werden dabei Referenzdatensätze entstehen, die den Anforderungen des FRBR-Modells ebenso wie musikwissenschaftlichen Ansprüchen gerecht werden und als Ausgangspunkt für etwaige konzeptionelle Erweiterungen für Musikwerke anderer Epochen und/oder Kulturkreise bereitstehen. Neben diese qualitative Bereicherung der GND gesellt sich die quantitative: Projekte wie die oben erwähnte Konzertdatenbank aber auch das Musikverlagsprojekt widmen sich einer Breite musikalischen Lebens, das quantitativ vermutlich gerade nicht von den ,großen Namen“ bestimmt wurde. Für eine Verknüpfung der Werke mit der GND müssten dort deshalb viele Werksätze angelegt oder qualifiziert werden. Die GND profitiert dabei

29 https://www.bach-digital.de/content/index.xed. 
nicht nur durch ihren wachsenden Umfang - auch inhaltlich würde sie durch eine größere Vielfalt ergänzt, böte umfangreiches Material für eine Kulturgeschichte neben der bisher etablierten und öffnete schließlich vielleicht sich selbst den Blick für auch andere Musiken neben der zunächst und bisher fokussierten sogenannten „ernsten“ Musik europäischer Herkunft der vergangenen fünf Jahrhunderte. Denn, im Wesentlich basierend auf den Musikalienbeständen großer Bibliotheken, reproduzieren Normdaten, wie sie in der GND für musikalische Werke enthalten sind, eine klar begrenzte Vorstellung vom musikalischen Kunstwerk. Im Modell sind Anforderungen „musikalischer Werke“ anderer geografischer oder inhaltlicher Kulturräume bisher nicht berücksichtigt. Sich dieser (derzeitigen) Grenzen der GND - wie jeder Normierung - bewusst zu sein, bietet dennoch die Chance, das Modell auf den beschränkten Rahmen „Ausschnitte der europäischen Kunstmusik des 15.-20. Jahrhunderts“ anzuwenden und innerhalb dieses streng abgegrenzten Korpus Erkenntnisse zu gewinnen oder es um integrierende Möglichkeiten weiterzuentwickeln.

\section{Fazit}

So bleibt festzuhalten: Die Erfassung musikalischer Werktitel in der GND bietet Potenzial über den rein identifizierenden Nutzen für bibliografische Zwecke hinaus.

Voraussetzung für eine Verwendung der Daten im Semantic Web sind neben den technischen Fragen - Format/Mapping, Schnittstellen/Bereitstellung, Storing der semantischen Daten - die konsistente und saubere Pflege der Daten in der GND. Für den Bereich der Musikwerke sind dazu noch einige konzeptionelle Entscheidungen und Verabredungen nötig. Projekte, die die Aufwertung und Qualifizierung der Daten im Blick haben, dienen dabei nicht nur der weiteren Datensammlung, sondern auch der Weiterentwicklung des Modells. Idealiter werden so die im Bibliotheksbereich erfassten Daten für kulturwissenschaftlich motivierte Fragen nachnutzbar gemacht und neue Erkenntnisse ermöglicht.

Die Nachnutzung musikalischer Werknormdaten der GND dient drei wesentlichen Funktionen sowohl für bibliothekarische als auch für wissenschaftliche Szenarien: Durch das Einbinden der GND-Informationen können die Entitäten der eigenen Datenbank identifiziert werden, sie werden durch die kontextualisierenden Informationen angereichert und schließlich über die GND-Verknüpfung selbst anschlussfähig für andere Projekte. Die musikalischen Werknormdaten der GND bieten auf diese Weise ein Scharnier, das Informationen unterschiedlicher Perspekti- ven kombiniert und in einem Wissensraum zugänglich macht.

Das Chalumeau als besetztes Instrument wird bisher nur für sehr wenige Fälle in der GND angegeben: in Kompositionen von Gregor Joseph Werner über Jan Dismas Zelenka und Telemann zu Graupner, mithin vom frühen bis zum mittleren 18. Jahrhundert. Eine für weitere Analysen nutzbare Datenbasis ergibt diese Handvoll Ergebnisse nicht. Die Vorstellung aber, dass die GND als zentrale Sammelstelle für Informationen zu musikalischen Werken konsequent eingesetzt würde, lässt hoffen, dass sich dieser Befund zu einem anderen Zeitpunkt ganz anders darstellt dass sich dann womöglich zeigt, dass das Chalumeau ähnlich wie der Zink, der im 19. Jahrhundert wieder auftaucht - häufiger an unerwarteter Stelle eingesetzt wurde, kombiniert mit bestimmten Gattungen oder bevorzugten Instrumentalpartnern und Erkenntnisse ermöglicht, die durch das direkte Quellenstudium nur mit vielfachem Aufwand hätten erreicht werden können. Eine Art Makroperspektive könnte die Mikro-Musikgeschichte auf diese Weise ergänzen und um neue Fragen bereichern. ${ }^{30}$

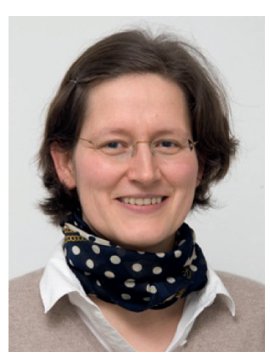

\section{Katrin Bicher}

Sächsische Landesbibliothek - Staats- und Universitätsbibliothek Dresden (SLUB) D-01054 Dresden katrin.bicher@slub-dresden.de

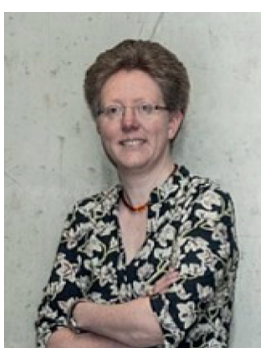

\section{Dr. Barbara Wiermann}

Sächsische Landesbibliothek - Staats- und Universitätsbibliothek Dresden (SLUB) D-01054 Dresden barbara.wiermann@slub-dresden.de

30 Nicht diskutiert wurde hier die Forderung einer unbedingten Quellenkritik, die im Metadatenbereich ebenso unabdingbar wie bei Primärdaten ist. Denn wie bei jeder Modellbildung muss auch im Bereich der Normdaten berücksichtigt werden, dass es sich um subjektive Reduktion von Informationen für einen bestimmten Zweck handelt. Vgl. auch Jannidis, Fotis; Kohle, Hubertus; Rehbein, Malte: Digital Humanities - Eine Einführung. Stuttgart 2017, 190, übertragen auf Metadaten. 